\title{
Double-diffusive convection in an inclined porous layer with a concentration-based internal heat source
}

Received: 12 April 2017 / Accepted: 11 August 2017 / Published online: 14 September 2017 (C) The Author(s) 2017. This article is an open access publication

\begin{abstract}
The thermosolutal instability of double-diffusive convection in an inclined fluid-saturated porous layer with a concentration-based internal heat source is investigated. The linear instability of small-amplitude perturbations to the system is analyzed with respect to transverse and longitudinal rolls. The resultant eigenvalue problem is solved numerically utilizing the Chebyshev tau method. It is shown that an increasing inclination angle causes a strong stabilization in the transverse rolls irrespective of the internal heat source or vertical solutal Rayleigh number. Furthermore, substantial qualitative changes are demonstrated in the linear instability thresholds with variations in the inclination angle and concentration-based heat source.
\end{abstract}

Keywords Inclined porous layer $\cdot$ Linear stability analysis $\cdot$ Heat source $\cdot$ Porous medium

\section{Introduction}

The investigation of thermosolutal convection driven by a concentration-based internal heat source with an inclined porous layer is vital due to its importance in real-life applications such as groundwater transportation, geothermal reservoirs, oil recovery, food processing and the transport of pollutants (Bendrichi and Shemilt [1], Chen and Angui [2]) and in more general environmental processes (Gill [3]).

The inclined porous layer was first studied by Bories and Combarnous [4] and later extended by Weber [5] and Caltagirone and Bories [6]. In more recent contributions, Rees and Bassom [7] investigated the thermal instability properties of Darcy flow in an inclined porous medium, highlighting the complex geometry of the neutral curves, and various asymptotic limits. The unicellular flow model in an inclined porous layer is explored in Barletta and Storesletten [8], with a constant heat flux boundary on the inclined porous channel further investigated by Rees and Barletta [9], and the thermal instability of Darcy Hadley flow in an inclined channel further explored by Barletta and Rees [10]. Nield [11] also commented on the inclined porous layer for preferred patterns of the natural convection, and thereafter, Nield et al. [12] studied the effect of the viscous dissipation influence on the onset of instability in an inclined porous channel. A collection of comprehensive theories on the inclined porous layer is surveyed in the recent book of Nield and Bejan [13]. Further surveys on porous media convection can be found in Ingham and Pop [14] and Vafai [15].

Communicated by Andreas Öchsner.

A. Matta

Department of Mathematics, FST, IFHE University, Dontanapalli, Hyderabad, Telangana 501504, India

E-mail: anjireddyiitm@gmail.com

A. A. Hill (ه)

Department of Applied Sciences, University of the West England, Bristol, UK

E-mail: antony.hill@uwe.ac.uk 
With more specific relation to recent work on internal heat sources, Parthiban and Patil [16] analyzed the thermal convection due to non-uniform heating boundaries with inclined thermal gradients in the presence of an internal heat source, followed by the extension to an anisotropic porous layer by Parthiban and Patil [17]. Rionero and Straughan [18] and Alex and Patil [19] examined the effect of variable gravity and an internal heat source, with Hill [20] reporting on double-diffusive convection with a concentration-based heat source. Bhadauria [21] and Capone et al. [22] further analyzed double-diffusive convection in a fluid-saturated anisotropic porous medium with an internal heat source. More recently, an inclined porous layer with an internal heat source was analyzed by Barletta et al. [23], where the upper wall was isothermal and the lower wall was either isothermal or adiabatic.

The main aim of this study is to analyze the stability of thermosolutal convection within an inclined porous layer including the effect of a concentration-based heat source. The externally imposed thermal gradient and heat source lead to a possibly unstable thermosolutal stratification in the inclined porous layer. The solutions of the linear instability analysis reduce to generalized eigenvalue problems which have been derived numerically using the Chebyshev tau technique [24].

\section{Mathematical analysis}

An infinite inclined porous layer saturated by a fluid with thickness $H$ is considered. The inclination angle of the porous layer along the $x^{*}$-axis is denoted by $\phi$, where the $z^{*}$-axis is chosen to be vertically upward. A physical representation of the model is given in Fig. 1.

The flow in the porous layer is described by Darcy's law (where the linear Boussinesq approximation is applicable) such that

$$
\frac{\mu}{K} \mathbf{q}^{*}+\nabla^{*} P^{*}=-\rho g\left(\sin (\phi) \mathbf{e}_{1}+\cos (\phi) \mathbf{e}_{3}\right)
$$

where $\mathbf{q}^{*}=\left(u^{*}, v^{*}, w^{*}\right)$ is the Darcy velocity, $P^{*}$ is the pressure, $g$ is acceleration due to gravity, $\mu$ is viscosity, $K$ is permeability with the vectors $\mathbf{e}_{1}$ and $\mathbf{e}_{3}$ being $(1,0,0)$ and $(0,0,1)$, respectively. Denoting the temperature to be $\theta^{*}$ and concentration to be $C^{*}$, we assume that the density of the fluid $\rho$ has a linear temperature and concentration dependence of the form

$$
\rho\left(\theta^{*}, C^{*}\right)=\rho_{f}\left(1-\gamma_{\theta}\left(\theta^{*}-\theta_{0}\right)+\gamma_{c}\left(C^{*}-C_{0}\right)\right)
$$

where $\rho_{f}, \theta_{0}$ and $C_{0}$ are reference values of density, temperature and concentration, respectively, and $\gamma_{\theta}$ and $\gamma_{C}$ are the coefficients for thermal and solutal expansion.

Combining (1) and (2) with the incompressibility condition and equation of energy and solutal balance yields the governing system

$$
\nabla^{*} \cdot \mathbf{q}^{*}=0
$$

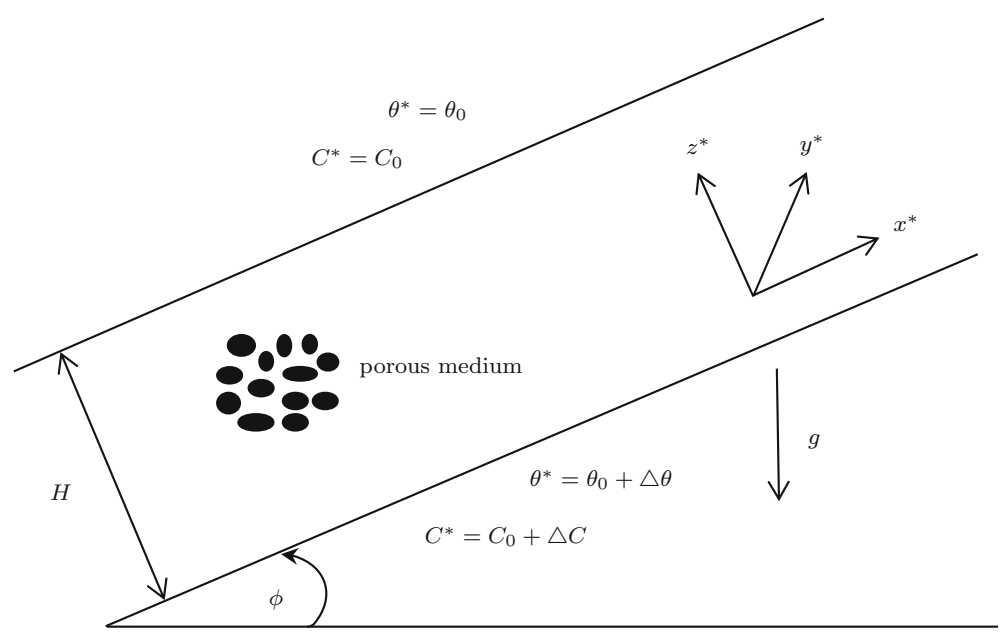

Fig. 1 Schematic diagram of the physical system 


$$
\begin{gathered}
\frac{\mu}{K} \mathbf{q}^{*}+\nabla^{*} P^{*}=-\rho_{f} g\left[1-\gamma_{\theta}\left(\theta^{*}-\theta_{0}\right)+\gamma_{c}\left(C^{*}-C_{0}\right)\right]\left(\sin (\phi) \mathbf{e}_{1}+\cos (\phi) \mathbf{e}_{3}\right), \\
\left(\rho_{f} c\right)_{m}\left(\frac{\partial \theta^{*}}{\partial t^{*}}\right)+\left(\rho_{f} c_{p}\right)_{f} \mathbf{q}^{*} \cdot \nabla^{*} \theta^{*}=k_{m} \nabla^{*^{2}} \theta^{*}+Q^{*}\left(C^{*}-C_{0}\right) \\
\Phi\left(\frac{\partial C^{*}}{\partial t^{*}}\right)+\mathbf{q}^{*} \cdot \nabla^{*} C^{*}=D_{m} \nabla^{*^{2}} C^{*}
\end{gathered}
$$

with the following boundary conditions:

$$
\begin{aligned}
& z^{*}=0: \quad w^{*}=0, \quad \theta^{*}=\theta_{0}+\triangle \theta \quad C^{*}=C_{0}+\triangle C \\
& z^{*}=H: \quad w^{*}=0, \quad \theta^{*}=\theta_{0} \quad C^{*}=C_{0}
\end{aligned}
$$

Here $\Phi$ is the porosity of the porous layer, $c$ denotes the specific heat, $k_{m}$ is the thermal conductivity, and $D_{m}$ is the solutal conductivity. The internal heat source is modeled linearly with respect to concentration, which is represented by the introduction of the $Q^{*}\left(C^{*}-C_{0}\right)$ term in the heat equation, where $Q^{*}$ is some constant of proportionality. The subscripts $f$ and $m$ are referred to fluid and porous medium, respectively.

Let us now consider the basic steady-state solution of (3)-(7) of the form

$$
\mathbf{q}_{s}^{*}=\left(u_{s}^{*}(z), 0,0\right), \quad P_{s}^{*}\left(x^{*}, y^{*}, z^{*}\right), \quad \theta^{*}\left(z^{*}\right), \quad C_{s}^{*}\left(z^{*}\right) .
$$

Assuming there is no net flow in horizontal direction (such that $\int_{0}^{H} u\left(z^{*}\right) \mathrm{d} z^{*}=0$ ), the steady state is given by

$$
\begin{aligned}
u_{s}^{*}\left(z^{*}\right)= & \frac{K \rho_{f} g}{\mu}\left[\frac{\gamma_{\theta} Q^{*} H^{2} \triangle C}{24 k_{m}}\left(\frac{4\left(z^{*}\right)^{3}}{H^{3}}-\frac{12\left(z^{*}\right)^{2}}{H^{2}}+\frac{8 z^{*}}{H}-1\right)\right. \\
& \left.+\left(\triangle \theta \gamma_{\theta}-\triangle C \gamma_{c}\right)\left(\frac{1}{2}-\frac{z^{*}}{H}\right)\right] \sin (\phi), \\
\theta_{s}^{*}\left(z^{*}\right)= & \frac{Q^{*} H^{2} \triangle C}{6 k_{m}}\left(\frac{\left(z^{*}\right)^{3}}{H^{3}}-\frac{3\left(z^{*}\right)^{2}}{H^{2}}+\frac{2 z^{*}}{H}\right)+\theta_{0}+\Delta \theta-\frac{\triangle \theta z^{*}}{H}, \\
C_{s}^{*}(z)= & C_{0}+\triangle C-\frac{\triangle C z^{*}}{H}
\end{aligned}
$$

To assess the stability of the steady solution, a perturbation $(\overline{\mathbf{q}}, \bar{P}, \bar{\theta}, \bar{C})$ is introduced to this steady state, such that

$$
\mathbf{q}^{*}=\mathbf{q}_{s}^{*}+\overline{\mathbf{q}}, \quad P^{*}=P_{s}^{*}+\bar{P}, \quad \theta^{*}=\theta_{s}^{*}+\bar{\theta}, \quad C^{*}=C_{s}^{*}+\bar{C},
$$

with a non-dimensionalization with scalings of

$$
\begin{aligned}
\left(x^{*}, y^{*}, z^{*}\right) & =H(x, y, z), \quad t^{*}=\frac{a H^{2}}{\alpha_{m}} t, \quad \overline{\mathbf{q}}=\frac{\alpha_{m}}{H} \mathbf{q}, \quad \bar{P}=\frac{\mu \alpha_{m}}{K} P, \\
\bar{\theta} & =\theta \triangle \theta, \quad \bar{C}=C \triangle C .
\end{aligned}
$$

Substituting the perturbations and non-dimensionalized variables into system (3)-(7) we derive

$$
\begin{gathered}
\nabla \cdot \mathbf{q}=0, \\
\mathbf{q}+\nabla P=\left[R_{z} \theta-\frac{S_{z}}{L e} C\right]\left(\sin (\phi) e_{1}+\cos (\phi) e_{3}\right), \\
\frac{\partial \theta}{\partial t}+\mathbf{q} \cdot \nabla \theta+F(z) w+G(z) \frac{\partial \theta}{\partial x}=\nabla^{2} \theta+Q C, \\
\left(\frac{\Phi}{a}\right) \frac{\partial C}{\partial t}+\mathbf{q} \cdot \nabla C-w+G(z) \frac{\partial C}{\partial x}=\frac{1}{L e} \nabla^{2} C,
\end{gathered}
$$




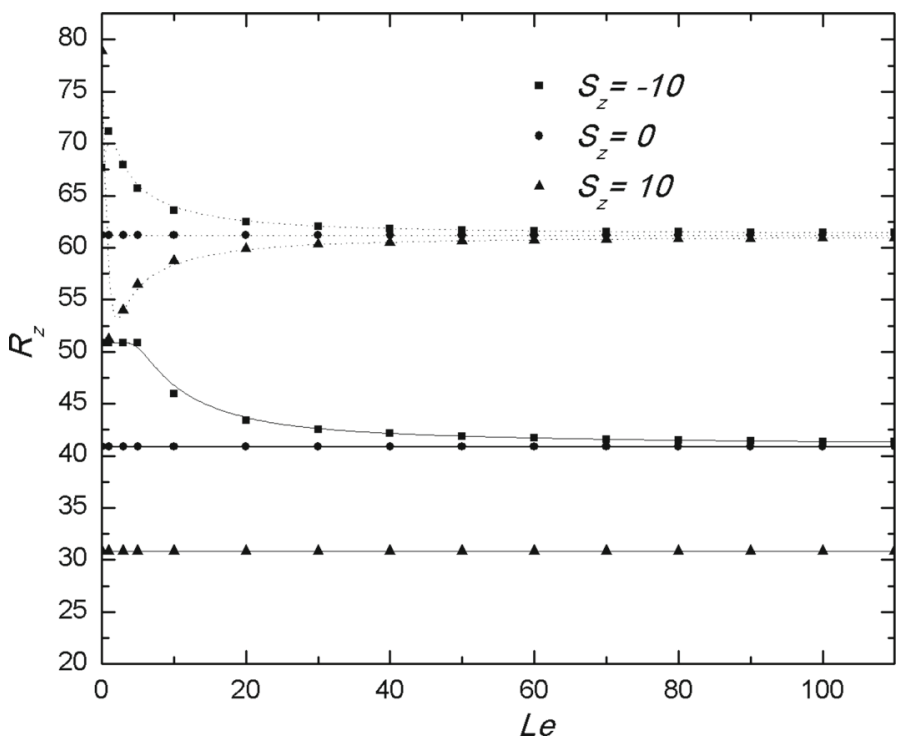

Fig. 2 Variation of $R_{z}$ with $L e$ at $\phi=15^{\circ}$ and $Q=0$

where

$$
\begin{gathered}
Q=\frac{H^{2} Q^{*} \triangle C}{k_{m} \triangle \theta}, \quad \alpha_{m}=\frac{k_{m}}{\left(\rho_{f} c_{p}\right)_{f}}, \quad a=\frac{\left(\rho_{f} c\right)_{m}}{\left(\rho_{f} c_{p}\right)_{f}}, \quad L e=\frac{\alpha_{m}}{D_{m}}, \\
R_{z}=\frac{\rho_{f} g \gamma_{\theta} K H \triangle \theta}{\mu \alpha_{m}}, \quad S_{z}=\frac{\rho_{0} g \gamma_{C} K H \triangle C}{\mu D_{m}},
\end{gathered}
$$

such that $R_{z}$ and $S_{z}$ are the vertical temperature and concentration Rayleigh numbers, respectively. Here

$$
\begin{gathered}
F(z)=\frac{Q}{6}\left(3 z^{2}-6 z+2\right)-1, \\
G(z)=\sin (\phi)\left[\frac{R_{z} Q}{24}\left(4 z^{3}-12 z^{2}+8 z-1\right)+\left(R_{z}-\frac{S_{z}}{L e}\right)(0.5-z)\right],
\end{gathered}
$$

with the boundary conditions

$$
\begin{array}{ccc}
z=0: & w=0, \quad \theta=1, \quad C=1 \\
z=1: & w=0, \quad \theta=0, \quad C=0 .
\end{array}
$$

\section{Linear stability analysis}

To proceed with the linear analysis, the nonlinear terms from (11) to (14) are discarded. As the resulting system is linear and autonomous, we may seek solutions of the form

$$
[\mathbf{q}, \theta, C, P]=[\mathbf{q}(z), \theta(z), C(z), P(z)] \exp \{i[k x+l y]+\sigma t\},
$$

where $k$ and $l$ are wave numbers in the $x$ and $y$ directions and $\sigma \in \mathbb{C}$ is the growth rate.

Letting $D=\frac{\mathrm{d}}{\mathrm{d} z}$ and taking the double curl of the linearized version of (12) where the third component is chosen (and the fact that $\mathbf{q}$ is solenoidal), we have the linearized system

$$
\begin{aligned}
\left(D^{2}-\alpha^{2}\right) w+ & \left(\alpha^{2} \cos (\phi)-k^{2} \sin (\phi)\right)\left[R_{z} \theta-\frac{S_{z}}{L e} C\right]=0, \\
\left(D^{2}-\alpha^{2}-i k G(z)\right) \theta-F(z) w+Q C & =\sigma \theta,
\end{aligned}
$$




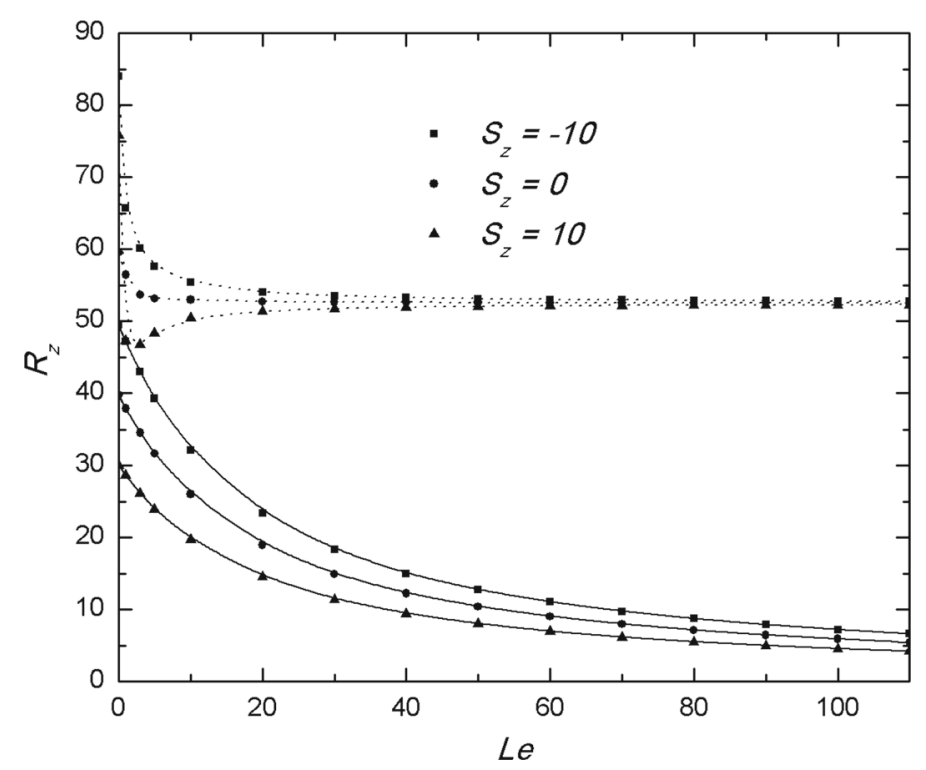

Fig. 3 Variation of $R_{z}$ with $L e$ at $\phi=15^{\circ}$ and $Q=1$

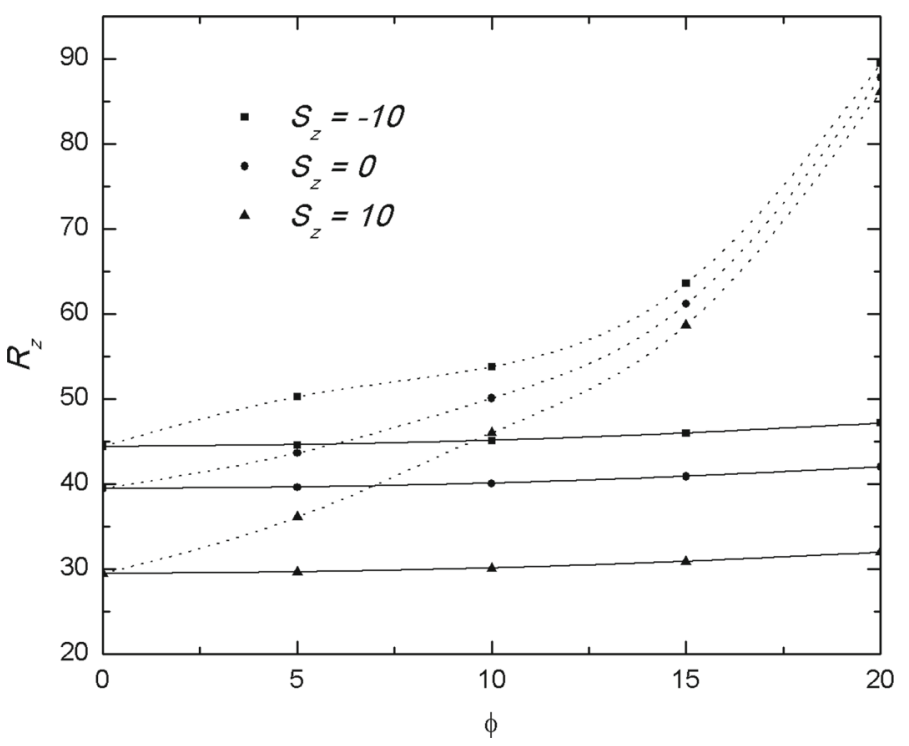

Fig. 4 Variation of $R_{z}$ with $\phi$ at $L e=10$ and $Q=0$

$$
\left(\frac{1}{L e}\left[D^{2}-\alpha^{2}\right]-i k G(z)\right) C+w=\sigma C .
$$

Equations (16)-(18) are subject to boundary condition (15), which gives an eigenvalue problem for a vertical thermal Rayleigh number $R_{z}$. Here, $\alpha=\sqrt{k^{2}+l^{2}}$ is the overall wave number. Eigenvalue problems (16)-(18) were solved by means of the Chebyshev tau method [24], where the results presented in Sect. 4.

\section{Results and discussion}

In this study the inclination angle $\phi$ is varied from $0^{\circ}$ to $20^{\circ}$, where we set $\Phi / a=1$ and $L e=10$ to represent a physical sugar or salt system. Here, the critical vertical thermal Rayleigh number $\left(R_{z}\right)$ is defined as the minimum of all $R_{z}$ values over the wave number $\alpha$. The critical values of transverse rolls are compared with the longitudinal rolls, where the longitudinal disturbances are characterized by $k=0$ and the transverse 


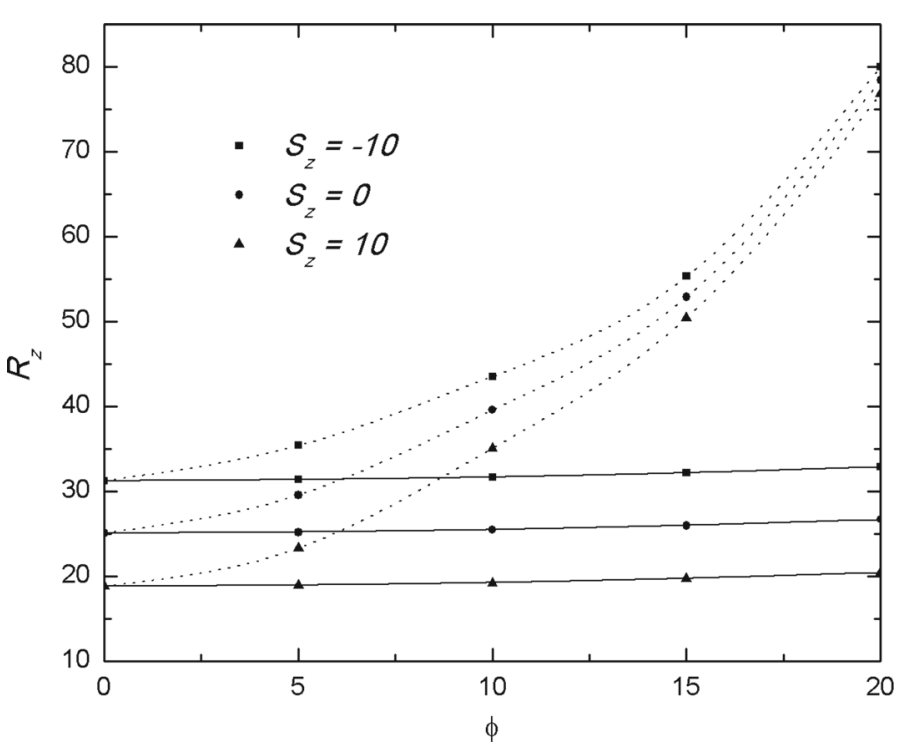

Fig. 5 Variation of $R_{z}$ with $\phi$ at $L e=10$ and $Q=1$

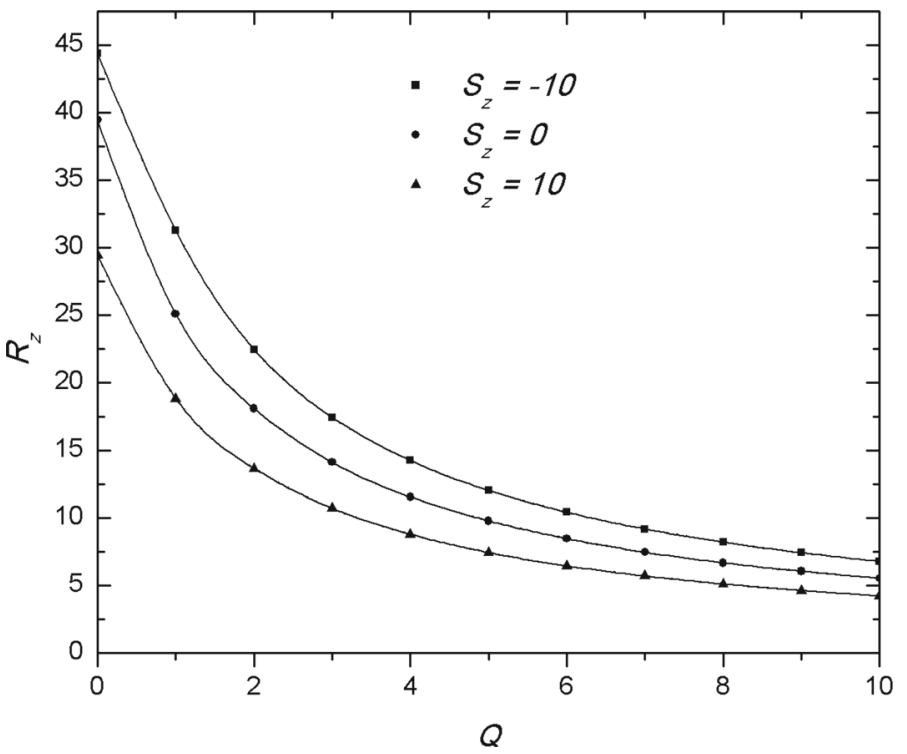

Fig. 6 Variation of $R_{z}$ with $Q$ at $L e=10$ and $\phi=0^{\circ}$

disturbances are characterized by $l=0$. Dotted lines represent the transverse roll results, and solid lines represent longitudinal roll results in Figs. 2, 3, 4, 5, 6, 7, 8 and 9.

Variations of $R_{z}$ as a function of $L e$, for various values of $S_{z}$ in both transverse and longitudinal rolls with inclination angle $\phi=15^{\circ}$, are given in Fig. 2 (absence of a concentration-based internal heat source $Q=0$ ) and Fig. 3 (presence of a concentration-based internal heat source $Q=1$ ). In both Figs. 2 and 3, the critical values of $R_{z}$ for the transverse rolls decrease up to approximately $L e=30$ for negative values of $S_{z}$, with insignificant variation thereafter. A similar behavior is demonstrated for positive values of $S_{z}$, although the critical values of $R_{z}$ increase (as oppose to decrease) up to approximately $L e=30$. In both Figs. 2 and 3, the critical values of $R_{z}$ for $S_{z}=0$ demonstrate minimal change with respect to a varying $L e$ for transverse rolls.

However, when considering longitudinal rolls, there is a considerable difference between the absence and presence of a concentration-based internal heat source. For $Q=0$ in Fig. 2 there is no variation in $R_{z}$ for $S_{z}=10$ and $S_{0}$ as $L e$ increases. However, for $Q=1$ in Fig. 3, there is a clear destabilization (represented by 


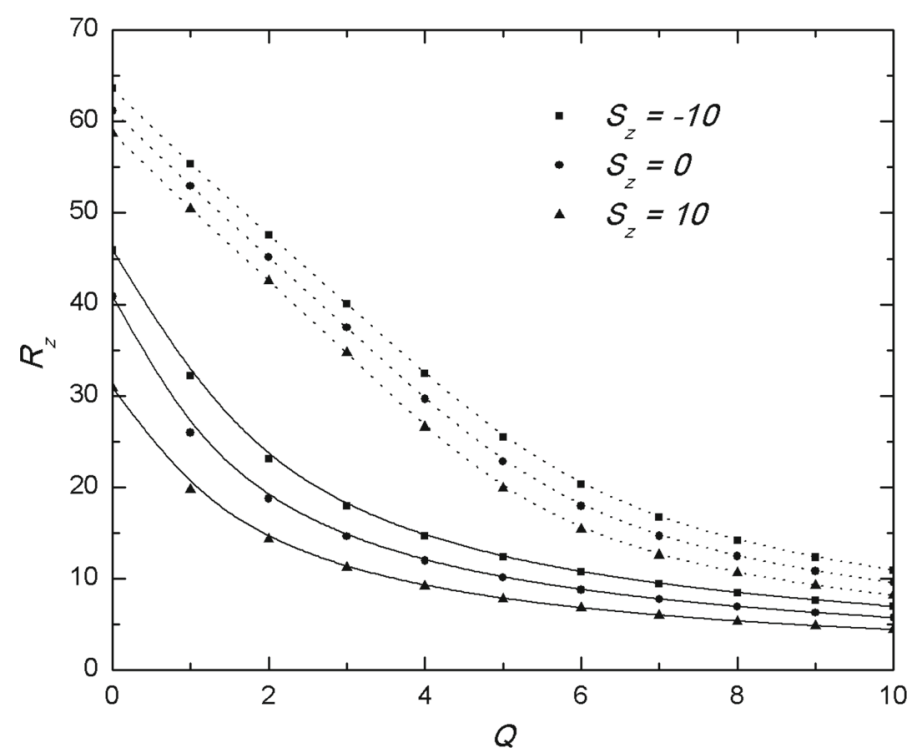

Fig. 7 Variation of $R_{z}$ with $Q$ at $L e=10$ and $\phi=15^{\circ}$

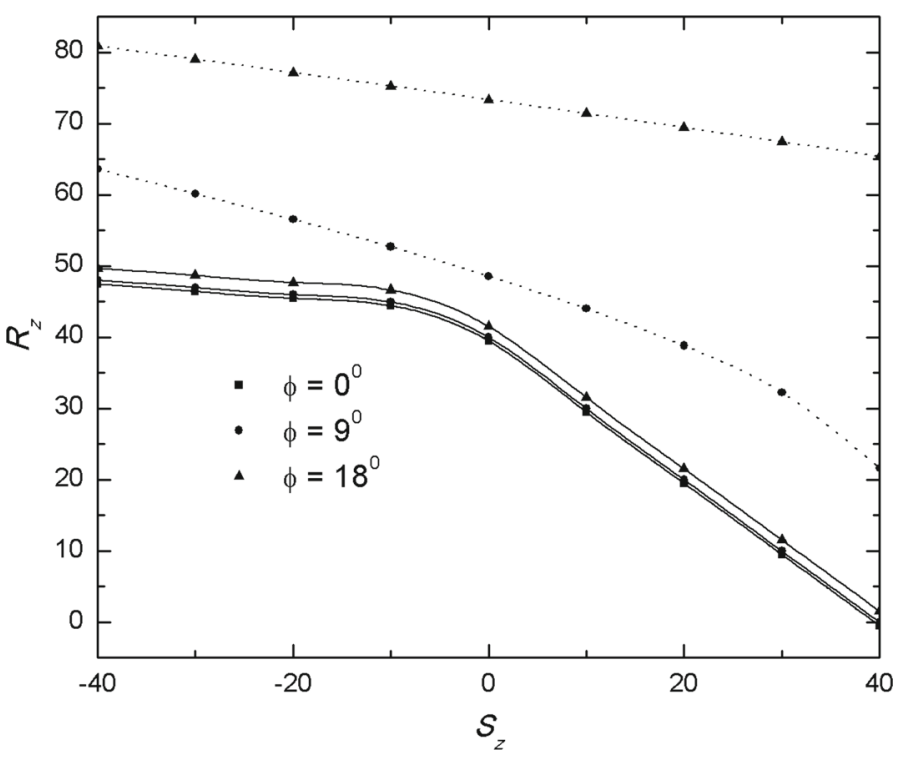

Fig. 8 Variation of $R_{z}$ with $S_{z}$ at $L e=10$ and $Q=0$

a decreasing $R_{z}$ ) as $L e$ increases. For $S_{z}=-10$ the destabilization caused by an increasing $L e$ is significantly more pronounced for $Q=1$ in Fig. 3 than for $Q=0$ in Fig. 2 .

The variation of critical values of $R_{z}$ as a function of inclination angle $\phi$ is given in Figs. 4 and 5, for the absence and presence of a concentration-based heat source, respectively, with different combinations of $S_{z}$. As the inclination angle $\phi$ is increased from $0^{\circ}$ to $20^{\circ}$, the critical values of $R_{z}$ increase in both the transverse and longitudinal rolls, although the effect is much more pronounced in the transverse rolls. Similar quantitative behavior is demonstrated for varying $\phi$ in both the absence (Fig. 4) and presence (Fig. 5) of a concentrationbased internal heat source for all values of $S_{z}$, although the presence of heat source is more destabilizing than without. In all the above observations, the transverse rolls are more stable than the longitudinal rolls.

A visual representation of $R_{z}$ as a function of concentration-based internal heat source $(Q)$ in both the absence and presence of inclination angle $\phi$ is shown in Figs. 6 and 7, respectively. In the absence of an inclination angle ( $\phi=0$ i.e., porous layer is horizontal), the critical values of $R_{z}$ for transverse and longitudinal rolls coincide, where the critical $R_{z}$ values decrease (i.e., the system becomes more unstable) with an increasing 


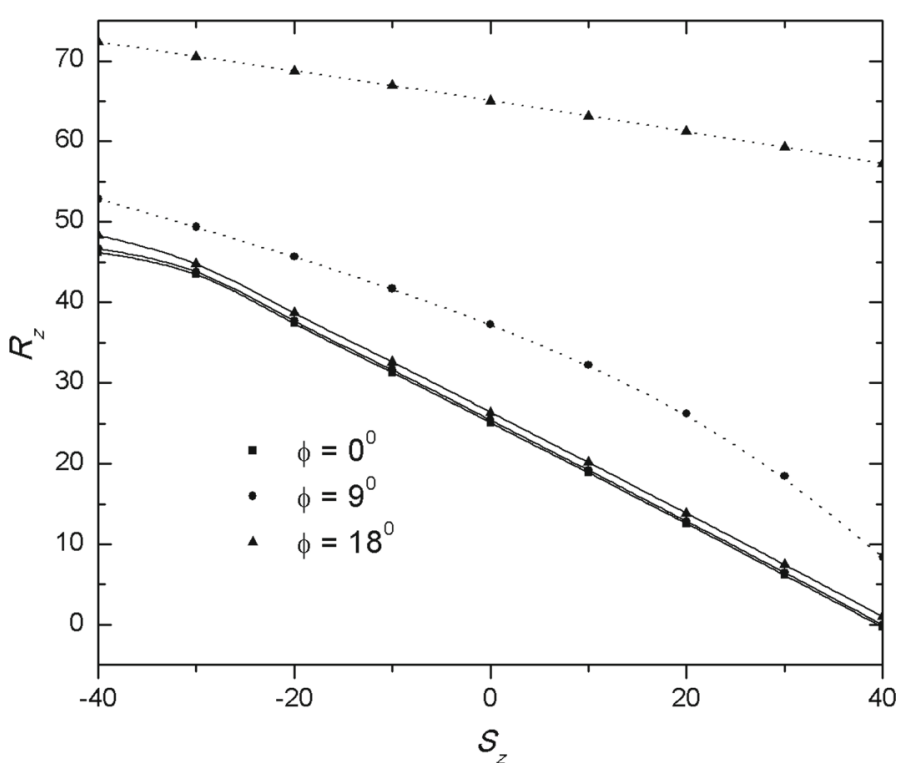

Fig. 9 Variation of $R_{z}$ with $S_{z}$ at $L e=10$ and $Q=1$

heat source for all values of $S_{z}$ (as shown in Fig. 6). It is interesting to observe that in the presence of inclination angle ( $\phi>0$ i.e., an inclined porous layer), the critical values of $R_{z}$ for transverse and longitudinal rolls differ more substantially at lower values of $Q$ than at higher values of $Q$ as shown in Fig. 7. Similarly to Fig. 6, the critical $R_{z}$ values decrease (i.e., the system becomes more unstable) with an increasing heat source for all values of $S_{z}$ in Fig. 7 .

The variation of $R_{z}$ with vertical solutal Rayleigh number $S_{z}$ in the absence and presence of a concentrationbased internal heat source is shown in Figs. 8 and 9, respectively, with different combinations of inclination angles $\phi$. The critical values of the transverse and longitudinal rolls show that an increase in the vertical solutal Rayleigh number destabilizes the system for both $Q=0$ and $Q=1$. In both Figs. 8 and 9, the longitudinal roll is more unstable than the transverse ones. Similarly to Fig. 8 , the critical $R_{z}$ values decrease (i.e., the system becomes more unstable) with an increasing heat source for all values of $S_{z}$ in Fig. 9. In all the above observations, the transverse rolls are more stable than the longitudinal rolls.

\section{Conclusion}

The onset of double-diffusive convection in an inclined fluid-saturated porous medium in the presence of concentration-based internal heat source is analyzed, where the thermosolutal instability analysis is explored through transverse and longitudinal rolls. The results demonstrate that:

- an increase in the concentration-based internal heat source causes strong destabilization in both the transverse and longitudinal rolls;

- in the absence of an inclination angle, the instability thresholds for the transverse and longitudinal rolls coincide;

- an increasing inclination angle causes a strong stabilization in the transverse rolls irrespective of the internal heat source or vertical solutal Rayleigh number;

- the critical $R_{z}$ values are higher in transverse rolls as compared to longitudinal rolls irrespective of the internal heat source and inclination angle;

- the critical $R_{z}$ values decrease as $S_{z}$ increases irrespective of the heat source and inclination angle;

- overall, substantial qualitative changes appear in the critical Rayleigh number subject to variations in the inclination angle and heat source.

Open Access This article is distributed under the terms of the Creative Commons Attribution 4.0 International License (http:// creativecommons.org/licenses/by/4.0/), which permits unrestricted use, distribution, and reproduction in any medium, provided you give appropriate credit to the original author(s) and the source, provide a link to the Creative Commons license, and indicate if changes were made. 


\section{References}

1. Bendrichi, G., Shemilt, L.W.: Mass transfer in horizontal flow channels with thermal gradients. Can. J. Chem. Eng. 75, 1067-1074 (1997)

2. Chen, X., Angui, L.: An experimental study on particle deposition above near-wall heat source. Build. Environ. 81, 139-149 (2014)

3. Gill, A.E.: A proof that convection in a porous vertical slab is stable. J. Fluid Mech. 35, 545-547 (1969)

4. Bories, S.A., Combarnous, M.A.: Natural convection in a sloping porous layer. J. Fluid Mech. 57, 63-79 (1973)

5. Weber, J.E.: Thermal convection in a tilted porous layer. Int. J. Heat Mass Transf. 18, 474-475 (1975)

6. Caltagirone, J.P., Bories, S.: Solutions and stability criteria of natural convective flow in an inclined porous layer. J. Fluid Mech. 155, 267-287 (1985)

7. Rees, D.A.S., Bassom, A.P.: Onset of Darcy-Bnard convection in an inclined layer heated from below. Acta Mech. 144, 103-118 (2000)

8. Barletta, A., Storesletten, L.: Thermoconvective instabilities in an inclined porous channel heated from below. Int. J. Heat Mass Transf. 54, 2724-2733 (2011)

9. Rees, D.A.S., Barletta, A.: Linear instability of the isoflux Darcy-Bnard problem in an inclined porous layer. Transp. Porous Media 87, 665-678 (2011)

10. Barletta, A., Rees, D.A.S.: Linear instability of the Darcy-Hadley flow in an inclined porous layer. Phys. Fluids 24, 074104 (2012)

11. Nield, D.A.: A note on convection patterns in an inclined porous layer. Transp. Porous Media 86, $23-25$ (2011)

12. Nield, D.A., Barletta, A., Celli, M.: The effect of viscous dissipation on the onset of convection in an inclined porous layer. J. Fluid Mech. 679, 544-558 (2011)

13. Nield, D.A., Bejan, A.: Convection in Porous Media, 4th edn. Springer, New York (2013)

14. Ingham, D.B., Pop, I.: Transport Phenomena in Porous Media. Pergamon, Oxford (1998)

15. Vafai, K.: Handbook of Porous Media, 2nd edn. CRC Press, Boca Raton (2005)

16. Parthiban, C., Patil, P.R.: Effect of non-uniform boundary temperatures on thermal instability in a porous medium with internal heat source. Int. Commun. Heat Mass Transf. 22, 683-692 (1995)

17. Parthiban, C., Patil, P.R.: Thermal instability in an anisotropic porous medium with internal heat source and inclined temperature gradient. Int. Commun. Heat Mass Transf. 24, 1049-1058 (1997)

18. Rionero, S., Straughan, B.: Convection in a porous medium with internal heat source and variable gravity effects. Int. J. Eng. Sci. 28, 497-503 (1990)

19. Alex, S.M., Patil, P.R.: Effect of a variable gravity field on convection in an anisotropic porous medium with internal heat source and inclined temperature gradient. Trans. ASME J. Heat Transf. 124, 144-150 (2002)

20. Hill, A.A.: Double-diffusive convection in a porous medium with a concentration based internal heat source. Proc. R. Soc. A 461, 561-574 (2005)

21. Bhadauria, B.S.: Double-diffusive convection in a saturated anisotropic porous layer with internal heat source. Transp. Porous Media 92, 299-320 (2012)

22. Capone, F., Gentile, M., Hill, A.A.: Double-diffusive penetrative convection simulated via internal heating in an anisotropic porous layer with throughflow. Int. J. Heat Mass Transf. 54, 1622-1626 (2011)

23. Barletta, A., Celli, M., Nield, D.A.: Unstable buoyant flow in an inclined porous layer with an internal heat source. Int. J. Therm. Sci. 79, 176-182 (2014)

24. Dongarra, J.J., Straughan, B., Walker, D.W.: Chebyshev tau-QZ algorithm methods for calculating spectra of hydrodynamic stability problems. Appl. Numer. Math. 22, 399-434 (1996) 